\title{
Analyzing the Unscented Kalman Filter Robustness for Orbit Determination Through Global Positioning System Signals
}

\author{
Paula Cristiane Pinto Mesquita Pardal ${ }^{1}$, Hélio Koiti Kuga², Rodolpho Vilhena de Moraes
}

\begin{abstract}
The nonlinear unscented Kalman filter (UKF) is evaluated for the satellite orbit determination problem, using Global Positioning System (GPS) measurements. The assessment is based on the robustness of the filter. The main subjects for the evaluation are convergence speed and dynamical model complexity. Such assessment is based on comparing the UKF results with the extended Kalman filter (EKF) results for the solution of the same problem. Based on the analysis of such criteria, the advantages and drawbacks of the implementations are presented. In this orbit determination problem, the focus is to analyze UKF convergence behavior using different sampling rates for the GPS signals, where scattering of measurements will be taken into account. A second aim is to evaluate how the dynamical model complexity affects the performance of the estimators in such adverse situation. After solving the real-time satellite orbit determination problem using actual GPS measurements, through EKF and UKF algorithms, the results obtained are compared in computational terms such as complexity, convergence, and accuracy.
\end{abstract}

KEYWORDS: Orbit determination, Nonlinear Kalman filters, GPS measurements, Real time.

\section{INTRODUCTION}

This work points out the nonlinear unscented Kalman filter (UKF) robustness assessment for a real-time satellite orbit determination problem, using Global Positioning System (GPS) measurements. This evaluation is based on comparing the UKF performance with the extended Kalman filter (EKF) for different sampling rates of the measurement from GPS signals. One-second analysis takes into account the dynamical model complexity effects on the performance of the two estimation techniques. The main subjects for the comparisons between the estimators are: convergence speed, divergence occurrence, faults, and statistical shortcomings. Based on the analysis of such criteria, the advantages and drawbacks of each estimator are exhibited.

The orbit determination of an artificial satellite is done using real data from the GPS receivers. In the orbit determination process of artificial satellites, the nature of both the dynamic system and the measurements equations are nonlinear. As a result, here it is necessary to manage a fully nonlinear problem in which the disturbing forces as well as the measurements are not easily modeled. This orbit determination problem lies in estimating the variables that completely specify a satellite trajectory in the space, processing a set of information (in this case, pseudo-range measurement) related to such body. As far as this work is concerned, the more accurate GPS phase measurements are not used here, because the main goal is not the search for accuracy, but a comparison of performance under 
different sampling rates of the measurements from GPS. Furthermore, if carrier phase measurements were used, the ambiguity resolution algorithm or any other artifacts to overcome such hindrance could eventually mask the results, misguiding the conclusions.

A spaceborne GPS receiver is a powerful resource to determine orbits of artificial Earth satellites by providing many redundant measurements which ultimately yields high degree of the observability to the problem. The Topex/Poseidon (T/P) satellite is a nice example of using GPS for space positioning. Through an onboard GPS receiver, the pseudo-ranges (error corrupted distance from satellite to each of the tracked GPS satellites) can be measured and used to estimate the full orbital state.

The EKF is very likely the most widely used realtime estimation algorithm for nonlinear systems (Maybeck, 1982). However, the experience from the estimation community has shown that the EKF is difficult to implement, requiring some skill to get tuned since depends very much on the nearness of the initial conditions to the true values; and the linearity on the time scale of the filter working updates. Many of these difficulties arise from the linearization required by the EKF method. Specifically for the orbit estimation problem, under inaccurate initial conditions (Pardal et al., 2011) and scattered measurements, the EKF implementation can lead to unstable or diverging solutions. Therefore, there is a strong need for a method that is probably more accurate than linearization, but that does not be liable to neither the implementation nor additional computational costs of other higher order filtering schemes. To overcome this limitation, the unscented transform (UT) was developed as a technique to propagate mean and covariance information through nonlinear transformations. The UKF is one of the sigma-point Kalman filters (SPKF), a new family of estimators that claims to yield equivalent or better performance than the EKF and elegantly to extend to nonlinear systems, without the linearization steps (van der Merwe, 2004; Julier and Uhlmann, 1997, 2004). This family of algorithms presents a new approach to generalize the KF for nonlinear dynamics and observation models.

Assessment between EKF and UKF was studied before by these and other authors, with different focus. Soken and Hajiyev (2011) compared two different robust Kalman filtering algorithms: Robust EKF and Robust UKF for the case of measurement malfunctions. In both filters, by the use of defined variables named as measurement noise scale factor, the faulty measurements were taken into the consideration with a small weight and the estimations were corrected without affecting the characteristic of the accurate ones. Proposed robust KFs were applied for the attitude estimation process of a pico satellite and the results are compared. El-Sheimy et al. (2006) studied which Kalman filtering design works best for GPS and micro-electromechanical (MEMS) inertial systems, since both have complementary qualities that make integrated navigation systems more robust. Jose (2009) implemented an UKF for integrating inertial navigation system (INS) with GPS and compared the results with the EKF approach, in performance and robustness. In a loosely coupled integrated INS/GPS system, inertial measurements from an inertial measurement unit IMU (angular velocities and accelerations in body frame) were integrated by the INS to obtain a complete navigation solution and the GPS measurements were used to correct for the errors and avoid the inherent drift of the pure INS system. Pardal et al. (2011) compared between the EKF and the nonlinear SPKF for a real-time satellite orbit determination problem, using GPS measurements for degraded initial conditions. The main subjects for the comparison between the estimators are convergence speed and computational implementation complexity. The aim was: to analyze the filters robustness; and to know the way such inaccuracies affect the performance of the estimators.

In this orbit determination problem the core is to analyze the convergence behavior for each filter in situations where there are different sampling rates of the measurement. Indeed there are small up to larger intervals between the processing of two GPS signals, and such intervals affect the performance of the estimators. A second goal evaluates the dynamical model complexity effects in the performance of the estimators for this case, especially during the adverse situations of larger reception intervals between two measurements. Therefore, the performance evaluation of the EKF (the most widely used estimation algorithm) and the UKF (supposedly the most appropriate estimation algorithm for nonlinear problems) to orbit determination problems in real time is due and justified. 


\section{SIGMA-POINT KALMAN FILTERS}

When the system dynamics and the observation model are of linear nature, the conventional KF is the optimal solution and must be used fearlessly. However, not rarely, the system dynamics and/or the measurement models are nonlinear, and convenient extensions of the KF, like the EKF, have been used.

The SPKF is a new family of estimators that allows similar performance to the KF for linear systems and elegantly extends to nonlinear systems, without need of the linearization procedures. This family of algorithm is a new approach to generalize the KF for nonlinear process and observation models (Julier and Uhlmann, 1997, 2004; van der Merwe et al., 2004). A set of weighted samples, the sigma-points, is used for computing mean and covariance of a probability distribution. Such algorithms include the UKF that is based on the UT, which is a nonlinear transformation of mean and covariance.

The SPKF represents a technique claimed as to lead to a more accurate and easier way to implement filter than the EKF or a second order Gaussian filter. Its approach is described, as follows (van der Merwe, 2004):

- A set of weighted samples is calculated deterministically based on the decomposition of the covariance and mean of a random variable.

- The sigma-points are propagated through the real nonlinear function, using only functional estimation, that is, analytical derivatives are not used to generate a posteriori set of sigma points.

- The later statistics are calculated using propagated sigma-points functions and weights. In general, they assume the form of a simple weighted average of the mean and the covariance.

Following, it will be separately explained the UT and the UKF, the filter stemming from this transformation.

\section{UNSCENTED TRANSFORM}

Essentially this is a manner of calculating the statistics of a random variable that passes through a nonlinear transformation. The UT approach is illustrated in Fig. 1 (Julier and Uhlmann, 1997; van der Merwe, 2004): select a suitable set of points (sigma-points) so that their mean and covariance are $\overline{\mathbf{x}}$ and $\mathbf{P}_{x x}$, respectively (Julier and Uhlmann, 1997, 2004). In turn, the

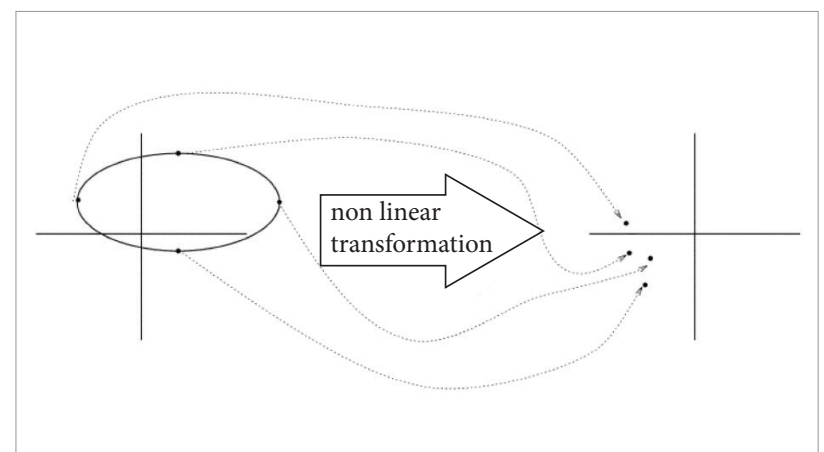

Figure 1. Unscented transform.

nonlinear function is applied to each point of the set to yield a cloud of transformed points. The statistics of the transformed points (mean $\overline{\mathbf{y}}$ and covariance $\mathbf{P}_{y y}$ ) can then be calculated to form an estimate of the nonlinearly transformed mean and covariance.

The sigma-points are carefully and deterministically chosen so that they exhibit certain specific properties, that is, they are not drawn at random like common Monte Carlo methods. Besides, they can be weighted in ways that are inconsistent with the distribution interpretation of sample points like in a particle filter (Julier and Uhlmann, 1997; van der Merwe, 2004).

The $n$-dimensional random variable $x$, with $\overline{\mathbf{x}}$ mean and $\mathbf{P}_{x x}$ covariance, is approximated by $2 n+1$ weighted points, the so known sigma-points, given by:

$\chi_{0}=\overline{\mathbf{x}}$

$\chi_{i}=\overline{\mathbf{x}}+\left(\sqrt{(n+\lambda) \mathbf{P}_{x x}}\right)$

$\chi_{i+n}=\overline{\mathbf{X}}-\left(\sqrt{(n+\lambda) \mathbf{P}_{x x}}\right)_{i}$

in which $\lambda=\alpha^{2}(n+k)-n$ includes scaling parameters. The constant parameter $\alpha$ controls the size of the sigma-points distribution $(0 \leq \alpha \leq 1)$, and $k$ provides an extra degree of freedom used to fine-tune the higher order moments; $k=3-n$ for a Gaussian distribution (Wan and van der Merwe, 2001). In Eq. 1, each element of the $n$-dimensional random variable $x$ is replaced by a set of sigma-points generated from the mean and the covariance of $x$. Thus, the vector variable $x$ has become a matrix of $n \times i$ dimension.

The transformation occurs as follows:

- Transform each point through the nonlinear function to yield the set of transformed sigma-points: 


$$
y_{i}=\mathbf{f}\left[\chi_{i}\right]
$$

- The observations mean is given by the weighted average of the transformed points:

$$
\overline{\mathbf{y}}=\sum_{i=0}^{2 n} W_{i} y_{i}
$$

- The covariance is the weighted outer product of the transformed points:

$\mathbf{P}_{y y}=\sum_{i=0}^{2 n} W_{i}\left[y_{i}-\overline{\mathbf{y}}\right]\left[y_{i}-\overline{\mathbf{y}}\right]^{T}$

$\mathrm{W}_{\mathrm{i}}$ is the weight associated to the $i$-th point given by:

$$
\begin{aligned}
& W_{0}=\frac{k}{(n+k)} \\
& W_{i}=\frac{1}{2(n+k)}, i=1, \ldots, n \\
& W_{i+n}=\frac{1}{2(n+k)}, i=1, \ldots, n
\end{aligned}
$$

\section{UNSCENTED KALMAN FILTER}

Using UT, the following steps are processed in the KF:

- Predict the new state system and its associated covariance, taking into account the effects of the Gaussian white noise process.

- Predict the expected observation and its residual innovation matrix considering the effects of the observation noise.

- Predict the cross correlation matrix.

In order to lead to the new filter, the UKF, these steps are arranged in the EKF, re-structuring: the dynamics; the state vector; and the observations model.

Table 1 presents an algorithm for the UKF. In the filter initialization, the mean, $\hat{\mathbf{x}}_{k-1}$, and the covariance matrix, $\hat{\mathbf{P}}_{k-1}$, of the state vector $\mathrm{x}$ are calculated, in reference to the prior instant, $t_{k-1}$. Following, the set of sigma-points is generated, from the mean and the covariance matrix, previously calculated. In the propagation step, the generated state sigma-point set is propagated to the instant $\mathbf{t}_{k}$, using the nonlinear dynamics equation (a), and the predict mean and covariance matrix are calculated (b). During the update cycle: the observations sigma-points are generated (a), propagated through the nonlinear observations equation (b), and its mean is obtained (c); the predict matrices of innovation, $\mathrm{P}_{k}^{v v}$, and correlation, $\mathrm{P}_{k}^{x y}$, are computed (d); and finally the Kalman gain is calculated, in order to update the state $\hat{\mathbf{x}}_{k}$, and the
Table 1. Unscented Kalman filter algorithm.

\section{Initialization $(\mathrm{t}=k-1)$ :}

$$
\begin{aligned}
& \hat{\mathbf{x}}_{k-1}=E\left[\mathbf{x}_{k-1}\right]=E\left[\mathbf{x}_{k-1}^{\mathrm{T}} \mathbf{w}_{k-1}^{\mathrm{T}}\right]^{\mathrm{T}} \in \mathfrak{R}^{n} \\
& \hat{\mathbf{P}}_{k-1}=E\left[\left(\mathbf{x}_{k-1}-\hat{\mathbf{x}}_{k-1}\right)\left(\mathbf{x}_{k-1}-\hat{\mathbf{x}}_{k-1}\right)^{\mathrm{T}}\right]
\end{aligned}
$$

\section{Sigma-points:}

$$
\begin{aligned}
& \boldsymbol{\chi}_{0, k-1}=\hat{\mathbf{x}}_{k-1} \\
& \boldsymbol{\chi}_{i, k-1}=\hat{\mathbf{x}}_{k-1}+\left(\sqrt{(n+\lambda) \hat{\mathbf{P}}_{k-1}}\right)_{i} ; \boldsymbol{\chi}_{i+n, k-1}=\hat{\mathbf{x}}_{k-1}-\left(\sqrt{(n+\lambda) \hat{\mathbf{P}}_{k-1}}\right)_{i}
\end{aligned}
$$

3. Propagation:

$$
\begin{array}{ll} 
& \boldsymbol{\chi}_{0, k}=\mathbf{f}\left[\boldsymbol{\chi}_{0, k-1}\right] \\
\text { a } & \boldsymbol{\chi}_{i, k}=\mathbf{f}\left[\boldsymbol{\chi}_{i, k-1}\right], i=1, \ldots, 2 n \\
\text { b } & \overline{\mathbf{x}}_{k}=\sum_{i=0}^{2 n} W_{i} \boldsymbol{\chi}_{i, k} \\
& \overline{\mathbf{P}}_{k}=\sum_{i=0}^{2 n} W_{i}\left[\boldsymbol{\chi}_{i, k}-\overline{\mathbf{x}}_{k}\right]\left[\boldsymbol{\chi}_{i, k}-\overline{\mathbf{x}}_{k}\right]^{\mathrm{T}}+\mathbf{Q}_{k}
\end{array}
$$

4. Update:

$$
\begin{array}{ll}
\mathrm{a} \quad \boldsymbol{\chi}_{i, k}=\overline{\mathbf{x}}_{k}+\left(\sqrt{(n+\lambda) \overline{\mathbf{P}}_{k}}\right)_{i} ; \boldsymbol{\chi}_{i+n, k}=\overline{\mathbf{x}}_{k}-\left(\sqrt{(n+\lambda) \overline{\mathbf{P}}_{k}}\right)_{i} \\
y_{0, k}=\mathbf{h}\left\lfloor\chi_{0, k}\right\rfloor \\
y_{i, k}=\mathbf{h}\left[\boldsymbol{\chi}_{i, k}\right], i=1, \ldots, 2 n \\
\mathrm{c} \quad \overline{\mathrm{y}}_{k}=\sum_{i=0}^{2 n} W_{i} y_{i, k} \\
\mathrm{~d} \quad \mathbf{P}_{k}^{v v}=\sum_{i=0}^{2 n} W_{i}\left[y_{i, k}-\overline{\mathrm{y}}_{k}\right]\left[y_{i, k}-\overline{\mathrm{y}}_{k}\right]^{\mathrm{T}}+\mathbf{R}_{k} \\
\mathbf{P}_{k}^{x y}=\sum_{i=0}^{2 n} W_{i}\left[\boldsymbol{\chi}_{i, k}-\overline{\mathbf{x}}_{k}\right]\left[y_{i, k}-\overline{\mathrm{y}}_{k}\right]^{\mathrm{T}} \\
\mathrm{e} \quad \hat{K}_{k}=\mathbf{P}_{k}^{x y}\left(\mathbf{P}_{k}^{v v}\right)^{-1} \\
\hat{\mathbf{x}}_{k}=\overline{\mathbf{x}}_{k}+\mathcal{K}_{k}\left(\mathbf{y}_{k}-\overline{\mathrm{y}}_{k}\right) \\
\hat{\mathbf{P}}_{k}=\overline{\mathbf{P}}_{k}-\mathcal{K}_{k} \mathbf{P}_{k}^{v v} \mathcal{K}_{k}^{\mathrm{T}}
\end{array}
$$

covariance matrix, $\hat{\mathbf{P}}_{k}$. They are used as a priori information in the next instant, $\mathbf{t}_{k+1}$, to generate the new set of sigma-points.

\section{EXTENDED KALMAN FILTER}

If the dynamical system and the observations model are linear, the $\mathrm{KF}$ is the recursive estimator most used at 
the present day since it is easy to implement and to use on digital computers. Its recursiveness leads to lesser memory storage, which makes it ideal for real-time applications. The EKF is a nonlinear version of the KF that generates reference trajectories which are updated at each measurement processing, at the corresponding instant (Maybeck, 1982; Brown and Hwang, 1985).

Because it is very difficult to accurately model the artificial satellites orbit dynamics, the EKF is generally used in works of such nature. Its algorithm always brings updated reference trajectory around the most current available estimate.

Exploiting the assumption that all transformations are quasi-linear, the EKF simply linearizes all nonlinear transformations and substitutes the Jacobian matrices for the linear transformations in the KF equations. The EKF consists of phases of time and measurement updates. In the first one, state and covariance are propagated from one precedent instant to a posterior one, which means that they are propagated between discrete instants of the system dynamics model. In the second one, state and covariance are corrected from the measurement obtained in the posterior instant of time, through the observations model. Therefore, the method nature is recursive, so it does not need to store previously the measurements in large matrices.

Following, the step for the EKF time update (or propagation) cycle is presented:

$\dot{\overline{\mathbf{x}}}_{k}=\mathbf{f}\left(\hat{\mathbf{x}}_{k-1}\right)$

$\overline{\mathbf{P}}_{k}=\varphi_{k, k-1} \hat{\mathbf{P}}_{k-1} \varphi_{k, k-1}^{T}+\mathbf{Q}_{k}$

where $\mathrm{f}$ is a nonlinear vector function modeling the orbit motion, $\overline{\mathbf{x}}_{k}$ and $\overline{\mathbf{P}}_{k}$ are respectively the propagated state and the covariance for $\mathrm{t}_{k} ; \boldsymbol{\varphi}_{k, k-1}$ is the state transition matrix between $\mathrm{t}_{k-1}$ e $\mathrm{t}_{k} ; \mathrm{Q}_{k}$ is the dynamics noise matrix given in Eq. 7. It is required the Jacobian matrix $(\partial \mathbf{f} / \partial \mathbf{x})$ for the transition matrix computation, which can be either simplified or very difficult to obtain.

$\mathrm{Q}_{k}=\int_{\mathrm{t}_{k-1}}^{\mathrm{t}_{k}} \varphi\left(\mathrm{t}, \mathrm{t}_{k-1}\right) \mathrm{G}(\mathrm{t}) \mathrm{Q}(\mathrm{t}) \mathrm{G}^{\mathrm{T}}(\mathrm{t}) \varphi^{\mathrm{T}}\left(\mathrm{t}, \mathrm{t}_{k-1}\right) \mathrm{dt}$

The equations for the EKF measurement update cycle are:

$$
\begin{aligned}
& \mathbf{K}_{k}=\overline{\mathbf{P}}_{k} \mathbf{H}_{k}^{T}\left(\mathbf{H}_{k} \overline{\mathbf{P}}_{k} \mathbf{H}_{k}^{T}+\mathbf{R}_{k}\right)^{-1} \\
& \hat{\mathbf{P}}_{k}=\left(\mathbf{I}-\mathbf{K}_{k} \mathbf{H}_{k}\right) \overline{\mathbf{P}}_{k} \\
& \hat{\mathbf{x}}_{k}=\overline{\mathbf{x}}_{k}+\mathbf{K}_{k}\left[\mathbf{y}_{k}-\mathbf{h}_{k}\left(\overline{\mathbf{x}}_{k}\right)\right]
\end{aligned}
$$

In Eq. 7, $\mathrm{G}(\mathrm{t})$ is the white noise addition matrix. In Eq. 8, $\mathbf{h}_{k}$ is a nonlinear vector function modeling the measurements; $\mathbf{H}_{k}$ is the corresponding partial derivative matrix $\left(\partial \mathbf{h}_{k} / \partial \mathbf{x}\right) ; \mathbf{K}_{k}$ is the Kalman gain; $\mathbf{R}_{k}$ is the observations noise matrix; $\hat{\mathbf{x}}_{k}$ and $\hat{\mathbf{P}}_{k}$ are respectively the state vector and the covariance updated for the instant $k ; \mathrm{y}_{\mathrm{k}}$ is the observations vector corresponding to the instant $k$.

Notwithstanding, the EKF has limitations. First: linearization can produce highly unstable filters if the assumptions of local linearity are violated; second: the derivation of the Jacobian matrices is nontrivial in most applications, and often leads to significant implementations difficulties (Julier and Uhlmann, 1997); third: analytical Jacobian matrices can be a very difficult and error-prone process.

Summarizing, linearization, as applied in the EKF, is widely recognized to be inadequate, but the alternatives incur substantial costs in terms of derivation and computational complexity. Hence, there is a strong need for a method that is probably more accurate than linearization but does not incur costs of implementation and computational of higher order than the other filters. The sigma-point algorithms were developed to meet these needs (Julier and Uhlmann, 1997).

\section{ORBIT DETERMINATION}

The orbit determination process consists of obtaining values of the parameters that completely specify the motion of an orbiting body (here, an artificial satellite), based on a set of observations of the body. It involves nonlinear dynamics and nonlinear measurement systems, which depends on the tracking system, and estimation technique (for instance, KF or least squares (Maybeck, 1982; Brown and Hwang, 1984)). The dynamical system model consists of the description for the dynamics of the satellite orbital motion, measurements models, Earth's rotation effects, and perturbation models. Furthermore the state variables defining the initial conditions, these models depend on a variety of parameters which affect both the dynamic motion as the measurement process (Montenbruck and Gill, 2001). Due to the complexity of the applied models it is hardly possible to solve such models equations directly for any of these parameters from a given set of observations. 
The observation may be obtained from the ground station networks using laser, radar, Doppler, or by space navigation systems, as the GPS. The choice of the tracking system depends on a compromise between the goals of the mission and the available tools. In the case of the GPS, the advantages are global coverage, high precision, low cost, and autonomous navigation resources. The GPS may provide orbit determination with accuracy at least as good as methods using ground tracking networks. The later provides standard precision around tens of meters and the former can provide precision as tight as some centimeters. The GPS provides, at a given instant, a set of many redundant measurements, which makes the orbit position observable geometrically.

After some advances of technology, the single frequency GPS receivers provide a good basis to achieve fair precision at relatively low cost, still attaining the accuracy requirements of the mission operation. The GPS allows the receiver to determine its position and time geometrically anywhere at any instant with data from at least four satellites. The principle of navigation by satellites is based in sending signals and data from the GPS satellites to a receiver located onboard the satellite that needs to have its orbit determined. This receiver measures the travel time of the signal and then calculates the distance between the receiver and the GPS satellite. If the clocks are not synchronized, four measurements are required to obtain its position. Those measurements of distances are called pseudo-ranges.

The instantaneous orbit determination using GPS satellites is based on the geometric method. In such method, the observer knows the set of GPS satellites position in a reference frame, obtaining its own position in the same reference frame.

However, sequential orbit determination makes use of the orbital motion modeling to predict between measurement times and measurement model to update the orbit by processing of measurements from GPS. This gives rise to recursive and real-time $\mathrm{KF}$ estimator for the orbit determination (Brown and Hwang, 1985).

\section{FILTER DYNAMIC MODEL}

In the case of orbit determination via GPS, the ordinary differential equations which represent the dynamic model are in its simplest form, given traditionally as follows:

$$
\begin{aligned}
& \dot{\mathrm{r}}=\mathrm{v} \\
& \dot{\mathrm{v}}=-\mu \frac{\mathrm{r}}{\mathrm{r}^{3}}+\mathrm{a}+\mathrm{w}_{v} \\
& \dot{\mathrm{b}}=\mathrm{d} \\
& \dot{\mathrm{d}}=0+\mathrm{w}_{\mathrm{d}}
\end{aligned}
$$

wherein the variables are placed in the inertial reference frame. In Eq. 9, $\mathbf{r}$ is the vector of the position components $(x, y, z) ; \mathbf{v}$ is the velocity vector; a represents the modeled perturbing accelerations; $\mathbf{w}_{v}$ is the white noise vector with covariance Q; b is the user satellite GPS clock bias; $d$, the user satellite GPS clock drift; and $\mathrm{w}_{\mathrm{d}}$ the noise associated with the GPS clock. The GPS receiver clock offset was not taken into account, so as not to obscure the conclusions drawn in this paper due to introduction of clock offset models in the filters. Indeed, the receiver clock offset was beforehand obtained and used to correct the GPS measurements, so that the measurements are free from the error derived from receiver clock offset.

\section{FORCE MODEL}

The main disturbing forces of gravitational nature that affect the orbit of an Earth's artificial satellite are: the nonuniform distribution of Earth's mass; ocean and terrestrial tides; and the gravitational attraction of the Sun and the Moon. There are also the non-gravitational effects, such as: Earth atmospheric drag; direct and reflected solar radiation pressure; electric drag; emissivity effects; relativistic effects; and meteorites impacts.

The disturbing effects are in general included according to the physical situation presented and to the accuracy that is intended for the orbit determination. Here, we include only a minimum set of perturbations which enable us to assess the performance of both filters, namely geopotential and third body point mass effect of Sun and Moon.

The Earth is not a perfect sphere with homogeneous mass distribution, and cannot be considered as a material point. Such irregularities disturb the orbit of an artificial satellite and the keplerian elements that describe the orbit do not behave ideally. The geopotential function can be given by (Kaula, 1966):

$U(\mathrm{r}, \phi, \lambda)=\frac{\mu}{r} \sum_{n=0}^{\infty} \sum_{m=0}^{n}\left(\frac{R_{\mathrm{T}}}{\mathrm{r}}\right)^{n} P_{n m}(\sin \phi)\left(C_{n m} \cos m \lambda+S_{n m} \sin m \lambda\right)$

where $\mu$ is Earth gravitational constant; $R_{T}$ is mean Earth radius; $r$ is the spacecraft radial distance; $\phi$ is the geocentric 
latitude; $\lambda$ is the longitude on Earth fixed coordinates system; $C_{n m}$ and $S_{n m}$ are the harmonic spherical coefficients of degree $n$ and order $m ; P_{n m}$ are the associated Legendre functions. The constants $\mu, R_{T}$, and the coefficients $C_{n m}$, and $S_{n m}$ determine a particular gravitational potential model.

Another gravitational perturbation source is due to the Sun and Moon attraction. They are more meaningful at larger distance from Earth. As the orbital variations are of the same type, be the Sun or the Moon the attractive body, they are normally studied without distinguishing the third body. The Sun-Moon gravitational attraction mainly acts on node and perigee, causing precession of the orbit and on the orbital plane. The general three-body problem model is here simplified to the circular restricted three-body problem, where the orbital motion of a third body (satellite), which mass can be neglected, around two other massive bodies is studied. The force acting on the third body (the satellite) in the inertial reference frame can be expressed as (Prado and Kuga, 2001; Guan, 2013):

$\dot{\mathbf{r}}_{3}=-G m_{1} \frac{\mathbf{r}_{13}}{\mathbf{r}_{13}^{3}}-G m_{2} \frac{\mathbf{r}_{23}}{\mathbf{r}_{23}^{3}}$

where $\mathbf{r}_{13}=\mathbf{r}_{3}-\mathbf{r}_{1}, \mathbf{r}_{23}=\mathbf{r}_{3}-\mathbf{r}_{2}$, and $\mathbf{r}_{i}, i=1,2,3$ corresponds to the $i$-th body distance vector to the center of mass of the system; and $m_{1}$ and $m_{2}$ are the masses of the Sun and the Moon, respectively.

\section{OBSERVATIONS MODEL}

The nonlinear equation of the observation model is:

$\mathbf{y}_{k}=\mathbf{h}_{k}\left(\mathbf{x}_{k}, \mathrm{t}\right)+v_{k}$

where, at time $t_{k}, \mathbf{y}_{k}$ is the vector of $m$ observations; $\mathbf{h}_{k}\left(\mathbf{x}_{k}\right)$ is the nonlinear function of state $\mathbf{x}_{k}$, with dimension $m$; and $v_{k}$ is the observations errors vector, with dimension $m$ and covariance $\mathbf{R}_{k}$. For the present application, one only uses the ion-free pseudo-range measurements from the GPS receiver of $\mathrm{T} / \mathrm{P}$ satellite. Also, the receiver clock offset was computed before and used to correct the pseudo-range measurements. In addition, the nonlinear pseudo-range measurement was modeled according to Chiaradia et al. (2003).

\section{MEASUREMENTS SAMPLING RATES IMPACT IN THE ORBIT DETERMINATION}

Previous presented studies (Pardal et al., 2009b, 2010 and Pardal, 2011) showed that the accuracy improvement for the dynamics models did not better the errors resulting between the references from precise orbit ephemeris from JPL/NASA (POE/JPL) and the values stemming from filters estimation process. This means that the magnitude of the errors obtained through the UFK or EKF is not reduced when increasing the complexity of the dynamic model: from a model for the geopotential with high degree and order to a complex model containing the three major disruptive effects of the orbit. Further, such results showed an equivalent competitiveness between the estimators, since the errors are of the same order of magnitude. It might have occurred because, in those results, the orbit determination process was done for small sampling intervals of the measurements.

Taking this into account, before concluding that a simpler dynamics modeling can be indiscriminately adopted, there is a need for another test (Pardal et al., 2010). Such test has two well determined purposes: to examine carefully the benefits of increasing the adopted dynamics model accuracy; and to investigate the apparent competitiveness between the estimators. This test rested in executing the orbit determination process considering different intervals between the GPS observations (pseudo-range) sampling. The intervals of sampling were 10, 30, $60,300,600,1200$, and 1800 seconds, in other words, conditions were swept from one very small range (10 seconds) to a range extremely high (1800 seconds) between the prediction and the subsequent correction of the predicted values to complete the cycle of the estimation process. With the gradual spacing of the interval between two measurements, the intention was to verify the dynamics models complexity, and the application of each filter in the time update cycle. In such situation, the propagation has it effects raised and the modeling accuracy becomes more significant in the accuracy of both filters estimative.

\section{RESULTS}

The tests and the analysis for the EKF and the UKF algorithms are presented. To validate and to analyze 
the methods, real GPS data from the T/P satellite are used. The filters estimated position and velocity are compared with T/P POE from JPL/NASA. The test conditions consider real ion-free pseudo-range data, collected by the GPS receiver onboard T/P, on November 19, 1993, at different sampling rates, presenting on average between 5 and 6 GPS satellites tracked. The GPS data were previously preprocessed to remove the outliers so they cannot mislead the filters or mask different data rejection policies of each filter. The tests have covered a long one day period of orbit determination.

The force model goes from a simple geopotential up to order and degree $(2 \times 2)$, with harmonic coefficients from JGM-2 model, to a model including perturbations due to geopotential up to order and degree $(28 \times 28)$ and due to the Sun-Moon gravitational attraction (Pardal et al., 2009a, 2010,
2011). The pseudo-range measurements were corrected to the first order with respect to ionosphere.

As already pointed, this work is not a search for results accuracy. It aims at UKF robustness assessment, which is done through the comparison of performance between UKF and EKF estimators under different sampling rates. There are peculiar interest for speed convergence, and divergence occurrence.

Table 2 shows the analysis for the predicted pseudo-range residuals convergence, which is measured in terms of time span, $\mathrm{Dt}_{\text {sam }}$, of data processed. The convergence is assumed when the residuals achieve similar statistics of the reference solution residuals. When small samplings intervals, such as 10,30 , and 60 seconds are used, convergence occurs instantaneously after the estimation process starts, for both UKF and EKF algorithms,

Table 2. Pseudo-range residuals convergence speed.

\begin{tabular}{|c|c|c|c|}
\hline \multirow{2}{*}{ Model } & \multirow{2}{*}{$\Delta$ tsam [s] } & UKF & EKF \\
\hline & & convergence time (h) & convergence time (h) \\
\hline \multirow{5}{*}{ Geo2 } & $10-30-60$ & 0 & 0 \\
\hline & 300 & no convergence observed & no convergence observed \\
\hline & 600 & - & - \\
\hline & 1200 & - & - \\
\hline & 1800 & - & - \\
\hline \multirow{5}{*}{ Geo5 } & $10-30-60$ & 0 & 0 \\
\hline & 300 & 0 & no convergence observed \\
\hline & 600 & no convergence observed & - \\
\hline & 1200 & - & - \\
\hline & 1800 & - & - \\
\hline \multirow{5}{*}{ Geo10 } & $10-30-60$ & 0 & 0 \\
\hline & 300 & 0 & no convergence observed \\
\hline & 600 & 0 & - \\
\hline & 1200 & 0 & - \\
\hline & 1800 & 2 & - \\
\hline \multirow{5}{*}{ Geo28 } & $10-30-60$ & 0 & 0 \\
\hline & 300 & 0 & 0 \\
\hline & 600 & 0 & 1.5 \\
\hline & 1200 & 0 & 5 \\
\hline & 1800 & 2 & no convergence observed \\
\hline \multirow{5}{*}{ S-M } & $10-30-60$ & 0 & 0 \\
\hline & 300 & 0 & 0 \\
\hline & 600 & 0 & 1.5 \\
\hline & 1200 & 0 & 5 \\
\hline & 1800 & 2 & no convergence observed \\
\hline
\end{tabular}


and for any dynamics model adopted. For this reason, results obtained for 10, 30, and 60 seconds of sampling interval will be placed in the same line of Table 1 . The model of geopotential up to low order and degree $2 \times 2$ (Geo2) starts diverging for 300 seconds of sampling interval, regardless of the estimator applied. The improved geopotential up to order and degree $5 \times 5$ (Geo5) dynamics model stops converging when UKF is the filter for 600 seconds, while EKF is not able to converge at all since 300 seconds. From an improved geopotential model and on, there is always occurrence of divergence when EKF is the algorithm, and convergence keeps occurring if UKF is the chosen algorithm. That is to say: for geopotential up to order and degree $10 \times 10$ (Geo10), divergence is detect at 300 seconds; and for geopotential up to high order and degree $28 \times 28$ (Geo28), and for a model that compounds geopotential up to order and degree 28 with Sun-Moon gravitational attraction (S-M), there is occurrence of divergence at 1800 seconds. The convergence time (consequently the convergence speed) is the same for the Geo28 and the S-M models up to 300 seconds of sampling interval, for both estimators, as shown in Table 1. The filters convergence time starts to be different at 600 seconds of interval between two measurements, and this difference keeps the same for each test case (600,1200, or 1800 seconds), whether the model is Geo28 or S-M. At this point it is possible to pinpoint a model limitation for convergence analysis.

Table 3 shows the convergence analysis for the position RNT (radial, normal, and along-track) components error, which is again measured in terms of data time of processing. When small intervals of sampling are used, such as 10, 30, and 60 seconds, convergence is detected instantaneously after the starts of the estimation process, for the two estimators, and for any choice of dynamics model. From 300 seconds on, the behavior is the same described in Table 2. The Geo 2 model errors start diverging for 300 seconds of sampling interval, regardless of the estimator applied. The improved Geo5 dynamics model errors stop converging when the UKF is the filter for 600 seconds, while

Table 3. Errors in position convergence speed.

\begin{tabular}{|c|c|c|c|c|c|c|c|}
\hline \multirow{2}{*}{ Model } & \multirow{2}{*}{$\Delta \mathrm{t}$ sam [s] } & \multicolumn{3}{|c|}{ UKF convergence time (h) } & \multicolumn{3}{|c|}{ EKF convergence time (h) } \\
\hline & & $\mathbf{R}$ & $\mathbf{N}$ & $\mathrm{T}$ & $\mathbf{R}$ & $\mathbf{N}$ & $T$ \\
\hline \multirow{5}{*}{ Geo2 } & $10-30-60$ & 0 & 0 & 0 & 0 & 0 & 0 \\
\hline & 300 & \multicolumn{3}{|c|}{ no convergence observed } & \multicolumn{3}{|c|}{ no convergence observed } \\
\hline & 600 & \multicolumn{3}{|c|}{-} & \multicolumn{3}{|c|}{ - } \\
\hline & 1200 & \multicolumn{3}{|c|}{-} & \multicolumn{3}{|c|}{-} \\
\hline & 1800 & \multicolumn{3}{|c|}{-} & \multicolumn{3}{|c|}{-} \\
\hline \multirow{5}{*}{ Geo5 } & $10-30-60$ & 0 & 0 & 0 & 0 & 0 & 0 \\
\hline & 300 & 0 & 0 & 0 & \multicolumn{3}{|c|}{ no convergence observed } \\
\hline & 600 & \multicolumn{3}{|c|}{ no convergence observed } & \multicolumn{3}{|c|}{-} \\
\hline & 1200 & \multicolumn{3}{|c|}{-} & \multicolumn{3}{|c|}{-} \\
\hline & 1800 & \multicolumn{3}{|c|}{ - } & \multicolumn{3}{|c|}{ - } \\
\hline \multirow{5}{*}{ Geo10 } & $10-30-60$ & 0 & 0 & 0 & 0 & 0 & 0 \\
\hline & 300 & 0 & 0 & 0 & \multicolumn{3}{|c|}{ no convergence observed } \\
\hline & 600 & 0 & 0 & 0 & \multicolumn{3}{|c|}{-} \\
\hline & 1200 & 0 & 0 & 0 & \multicolumn{3}{|c|}{ - } \\
\hline & 1800 & 1 & 1 & 0 & \multicolumn{3}{|c|}{ - } \\
\hline \multirow{5}{*}{ Geo28 } & $10-30-60$ & 0 & 0 & 0 & 0 & 0 & 0 \\
\hline & 300 & 0 & 0 & 0 & 0 & 0 & 0 \\
\hline & 600 & 0 & 0 & 0 & 0.5 & 0.2 & 0.3 \\
\hline & 1200 & 0 & 0 & 0 & 1 & 3.5 & 3.2 \\
\hline & 1800 & 1 & 1 & 0 & 9 & 9.3 & $\begin{array}{c}\text { no convergence } \\
\text { observed }\end{array}$ \\
\hline \multirow{5}{*}{ S-M } & $10-30-60$ & 0 & 0 & 0 & 0 & 0 & 0 \\
\hline & 300 & 0 & 0 & 0 & 0 & 0 & 0 \\
\hline & 600 & 0 & 0 & 0 & 0.5 & 0.2 & 0.3 \\
\hline & 1200 & 0 & 0 & 0 & 1 & 3.5 & 3.2 \\
\hline & 1800 & 1 & 1 & 0 & 9 & 9.3 & $\begin{array}{c}\text { no convergence } \\
\text { observed }\end{array}$ \\
\hline
\end{tabular}


the EKF is not able to converge at all since 300 seconds. For the Geo10 dynamic model, divergence is detected at 300 seconds; and for the Geo28, and S-M models, there is occurrence of divergence at 1800 seconds. Again, for models Geo28 and S-M, it can be noticed that filters convergence time difference starts at 600 seconds of sampling interval, and this difference keeps the same for each test case $(600,1200$, or 1800 seconds), no matter the model. So far, a model limitation for convergence analysis might be clear.

Another statistical check is done, in order to confirm that the algorithms effectively reached convergence. The reference pseudo-range residuals statistics (mean and standard deviation) for each model and filter are available in the yellow lines of Table 4. As the three most improved dynamics models (Geo10, Geo28, and S-M) statistics for 10, 30, and 60 seconds considerably resemble (Pardal et al., 2009b, 2010), "reference" in Table 4 refers to a 60 -s sampling interval, and is representative of the three intervals. Now, in the analysis of the two poorest dynamics models (Geo2 and Geo5), all the sampling intervals are explicit in Table 4 in order to show poor dynamics behavior as the sampling intervals increase. It is clear that poor dynamics models are more sensitive to the intervals enlargement, since Geo2 model stops converging at 300 seconds, and Geo5, at 300 or 600 seconds, depending on the estimator applied. Through Table 4 it is also noticeable that if the model is too poor (e.g., Geo2), neither UKF nor EKF are able to keep convergence for larger sampling intervals, and divergence behavior is detected at 300 seconds for both. However, if the model is slightly improved (for instance, Geo5), UKF implementation shows more robustness than EKF one, because still converges at 300 seconds, while EKF starts diverging. From Table 3, it becomes evident that the estimators really reached convergence, since their statistical values remain nearly the same as the reference ones.

In order to portray such findings, Fig. 2 illustrates the reference residuals (small sampling intervals of 60 seconds) behavior, and the 1800 seconds sampling interval case behavior for both the EKF and the UKF estimators, using S-M as the dynamics model. It clearly indicates clues of EKF's divergence for such larger sampling intervals of measurements.

Proceeding the investigation, Table 5 shows total Root Mean Square (RMS) position error, where the reference values are again listed in the first row (yellowed). Again, in the analysis of the two poorest dynamics models (Geo2 and Geo5), all the sampling intervals are registered in Table 5 in order to show poor dynamical behavior as the sampling

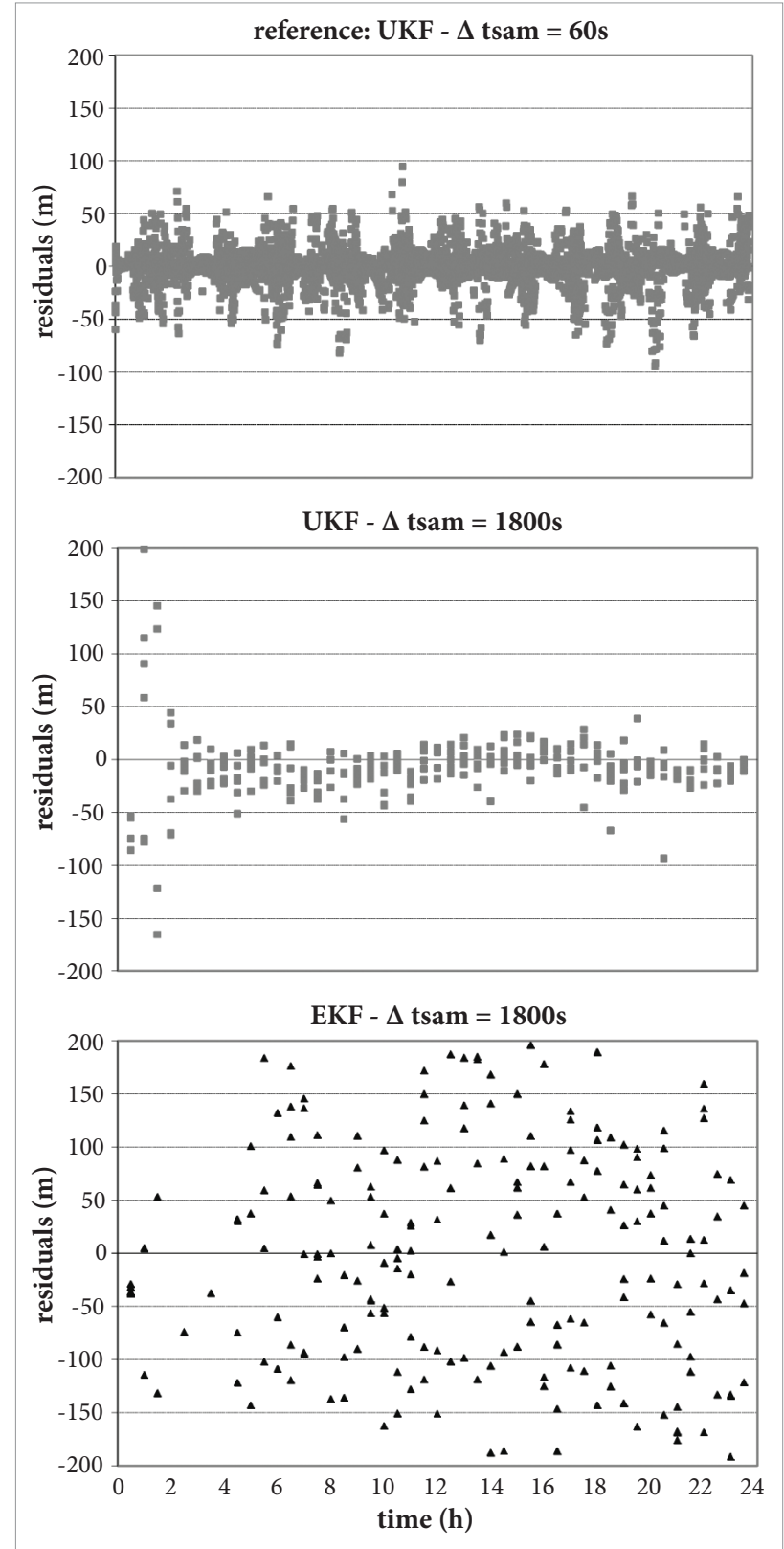

Figure 2. Pseudo-range residuals convergence and divergence occurrences.

intervals increase. It is clear that poor dynamics models are more responsive to the intervals increasing: the Geo 2 model results stop converging at 300 seconds while the Geo5, at 300 or 600 seconds, according to the estimator applied. Through Table 5 it is also perceptible that for an excessively poor model (Geo2, for example), UKF and EKF are unable to converge for larger sampling intervals, and divergence occurs at 300 seconds for both. However, if the model is slightly improved (for instance, Geo5), UKF implementation still 
converges at 300 seconds, while EKF starts diverging, which indicates more robustness of UKF when compared to EKF. UKF and EKF resulting RMS errors are only computed after assumed convergence time. For Table 5 , it is also verifiable that the estimators really reached convergence, since their RMS values remain nearly close to the reference ones.

Next, Fig. 3 depicts a particular case where a relatively poor Geo5 dynamics model is adopted. While the UKF filter

Table 4. Pseudo-range residual statistics, after convergence.

\begin{tabular}{|c|c|c|c|}
\hline \multirow{2}{*}{ Model } & \multirow{2}{*}{$\Delta$ tsam [s] } & UKF residuals & EKF residuals \\
\hline & & mean $\pm S D(m)$ & mean $\pm S D(m)$ \\
\hline \multirow{4}{*}{ Geo2 } & 10 & $0.218 \pm 18.504$ & $0.155 \pm 18.531$ \\
\hline & 30 & $0.081 \pm 26.992$ & $-0.003 \pm 27.103$ \\
\hline & 60 & $-0.091 \pm 37.687$ & $-0.276 \pm 37.916$ \\
\hline & 300 to 1800 & no convergence observed & no convergence observed \\
\hline \multirow{5}{*}{ Geo5 } & 10 & $0.138 \pm 13.064$ & $0.076 \pm 13.133$ \\
\hline & 30 & $-0.007 \pm 14.602$ & $-0.087 \pm 15.058$ \\
\hline & 60 & $-0.251 \pm 16.575$ & $-0.426 \pm 17.914$ \\
\hline & 300 & $-1.604 \pm 25.216$ & no convergence observed \\
\hline & 600 to 1800 & no convergence observed & - \\
\hline \multirow{5}{*}{ Geo10 } & reference & $-0.187 \pm 14.690$ & $-0.277 \pm 16.221$ \\
\hline & 300 & $-1.272 \pm 18.766$ & no convergence observed \\
\hline & 600 & $-2.401 \pm 19.786$ & - \\
\hline & 1200 & $-4.193 \pm 23.873$ & - \\
\hline & 1800 & $-7.714 \pm 33.229$ & - \\
\hline \multirow{5}{*}{ Geo28 } & reference & $-0.091 \pm 14.363$ & $-0.180 \pm 15.942$ \\
\hline & 300 & $-1.234 \pm 17.489$ & $1.861 \pm 45.886$ \\
\hline & 600 & $-2.379 \pm 18.242$ & $-3.557 \pm 27.425$ \\
\hline & 1200 & $-4.216 \pm 22.295$ & $-19.831 \pm 90.423$ \\
\hline & 1800 & $-7.929 \pm 32.163$ & no convergence observed \\
\hline \multirow{5}{*}{ S-M } & reference & $-0.109 \pm 14.021$ & $-0.201 \pm 15.639$ \\
\hline & 300 & $-1.294 \pm 15.872$ & $-1.021 \pm 43.197$ \\
\hline & 600 & $-2.397 \pm 16.542$ & $-3.6126 \pm 25.945$ \\
\hline & 1200 & $-4.163 \pm 20.518$ & $-19.814 \pm 91.237$ \\
\hline & 1800 & $-7.641 \pm 31.156$ & no convergence observed \\
\hline
\end{tabular}
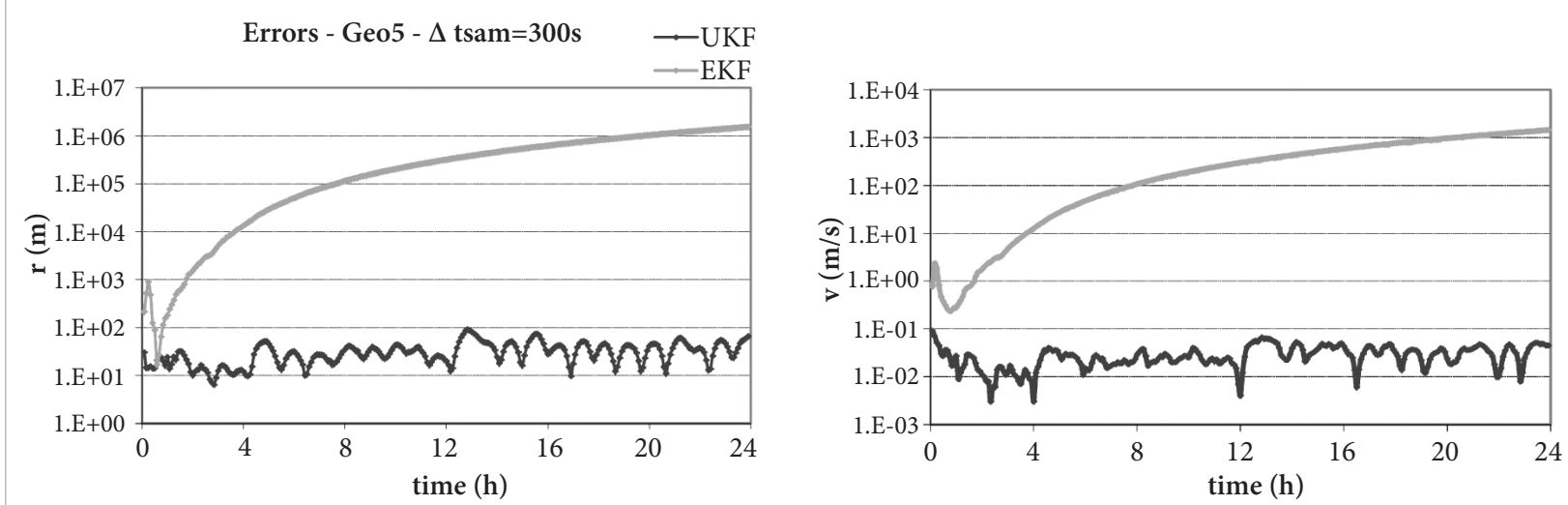

Figure 3. Convergence and divergence behavior for Geo5 dynamics model. 
Table 5. Error in position total Root Mean Square, after convergence.

\begin{tabular}{|c|c|c|c|}
\hline \multirow{2}{*}{ Model } & \multirow{2}{*}{$\Delta$ tsam [s] } & UKF & EKF \\
\hline & & total RMS[m] & total RMS[m] \\
\hline \multirow{4}{*}{ Geo2 } & 10 & 34.336 & 34.391 \\
\hline & 30 & 49.834 & 49.703 \\
\hline & 60 & 68.774 & 69.837 \\
\hline & 300 to 1800 & no convergence observed & no convergence observed \\
\hline \multirow{5}{*}{ Geo5 } & 10 & 20.521 & 20.642 \\
\hline & 30 & 20.050 & 20.301 \\
\hline & 60 & 22.481 & 27.303 \\
\hline & 300 & 35.562 & no convergence observed \\
\hline & 600 to 1800 & no convergence observed & - \\
\hline \multirow{5}{*}{ Geo10 } & reference & 18.150 & 23.953 \\
\hline & 300 & 22.273 & no convergence observed \\
\hline & 600 & 22.658 & - \\
\hline & 1200 & 24.000 & - \\
\hline & 1800 & 28.962 & - \\
\hline \multirow{5}{*}{ Geo28 } & reference & 17.135 & 23.159 \\
\hline & 300 & 19.217 & 21.798 \\
\hline & 600 & 19.386 & 29.487 \\
\hline & 1200 & 20.943 & no convergence observed \\
\hline & 1800 & 26.420 & - \\
\hline \multirow{5}{*}{ S-M } & reference & 16.253 & 22.520 \\
\hline & 300 & 15.437 & 18.416 \\
\hline & 600 & 15.398 & 26.489 \\
\hline & 1200 & 16.403 & no convergence observed \\
\hline & 1800 & 23.990 & - \\
\hline
\end{tabular}

remains converging, a divergence behavior is shown in the EKF implementation, for 300 seconds of sampling interval between two measurements. This result indicates that even if the model is not adequately chosen, the EKF believes that the model and linearization are correct. The UKF does not have linearizations and, in adverse situations, such as the ones of larger sampling intervals between two data samples or inaccurate initial conditions (Pardal et al., 2011), behaves more adequately. However, if the dynamics model is extremely truncated, such as the Geo2 analyzed in this work, neither the UKF nor the EKF will reach convergence for large sampling intervals, as shown in Tables 1-4. In Fig. 3, $\Delta \mathrm{r}$ and $\Delta \mathrm{v}$ represents, respectively, the absolute value of the errors in position and in velocity, in the inertial reference frame coordinates.

Figure 4 shows the errors in the RNT components for the UKF and EKF reference cases (small 60-s sampling interval, left side) and the larger 1800 seconds error case results for the
EKF and the UKF estimators (right side). The outstanding behavior in the right side happens again in any "no convergence observed" case pointed out in Table 4. It indicates signs of the EKF divergence for such a very large sampling interval, while UKF reaches the convergence zone, not much later than the left side results. So far, the results showed that the performance of the filters decreases as increasing the sampling intervals (according to assays of the error in position, the pseudorange residuals, and the convergence presented previously). These results point to the advantage of using the nonlinear theory for orbital dynamics, in small intervals of the UKF and the EKF algorithms.

In order to finish the results analysis, it is to be said that even considering the convergence for small sampling intervals, where UKF and EKF present similar performance, the algorithm is very sensitive to the initialization of the covariance matrix. This means that the algorithm convergence depends on the proper choice of such matrix. Therefore, the 


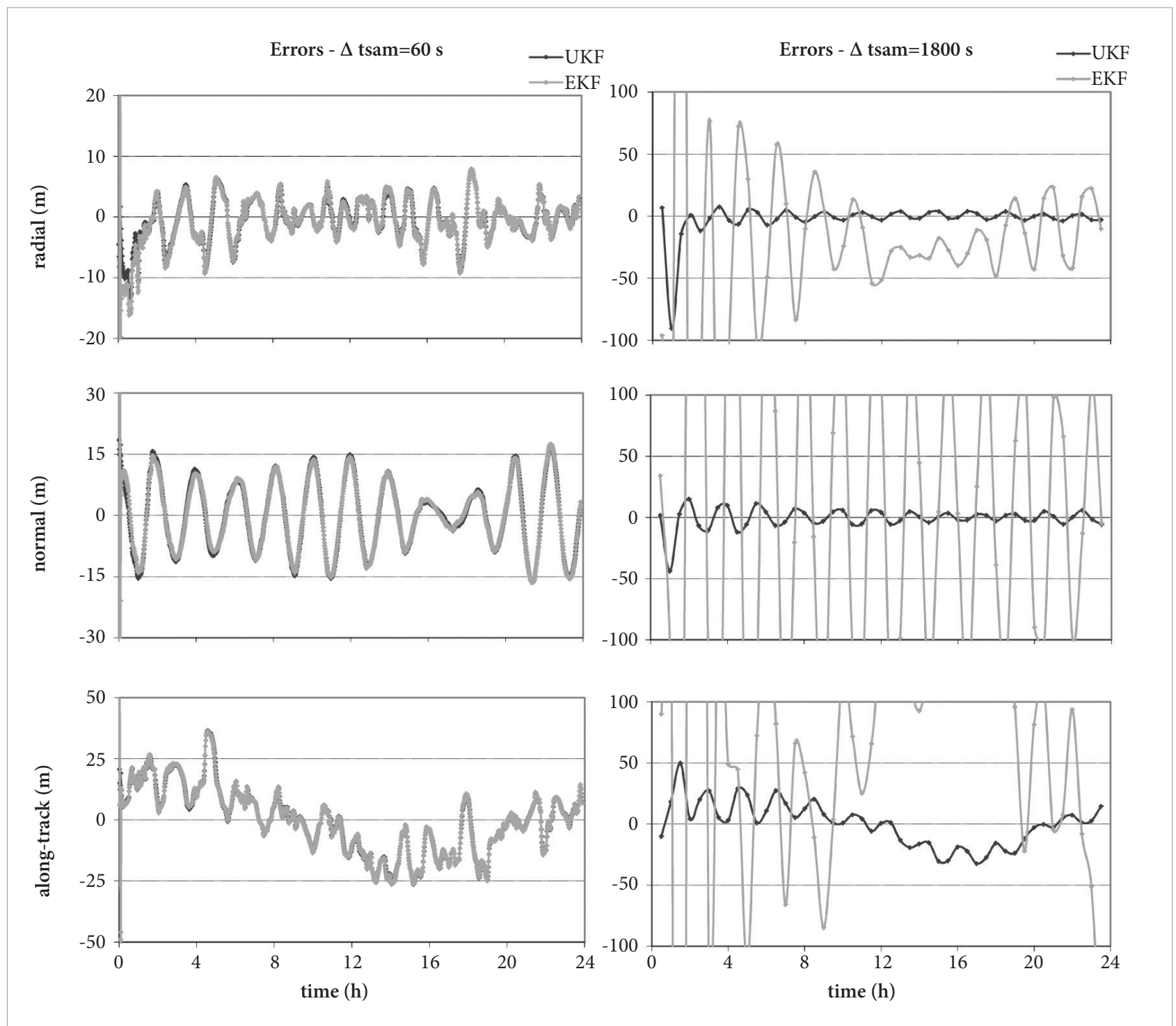

Figure 4. Convergence and divergence behavior of the errors in RNT (radial, normal, and along-track) components.

results are not general, and the algorithm was adjusted for this type of orbit determination application.

\section{CONCLUSIONS}

The robustness to increasing sampling intervals of two nonlinear estimators, namely the EKF and UKF was assessed for a real-time satellite orbit determination problem using real GPS measurements. One day (24h) of GPS receiver measurements of $\mathrm{T} / \mathrm{P}$ satellite at different sampling rates were processed. The emphasis was to characterize each filter convergence behavior in situations where the sampling rates vary from small to larger intervals between two measurements. Different dynamical models were analyzed, in order to establish the modeling effects in the orbit determination process.

Results showed that when small sampling intervals are used, UKF and EKF yield similar performance, with high performance, for almost all dynamics models. The exception is made if the model is extremely truncated (Geo2) or slightly improved (Geo5), where in the later Geo5 case, UKF maintains convergence and in the former Geo2 case, none of the estimators reach convergence even for 60-s sampling interval. As expected, increasing the sampling intervals decreases the 
filters performance. As larger is the interval more difficult is for EKF and UKF to reach convergence. When UKF is compared with EKF, in all cases of larger intervals, the UKF always attains convergence first. The rupture threshold for this application in particular occurs to all modeling complexities if EKF was the used algorithm. Only in two situations (the two poorest models adoption) for the UKF implementation, divergence occurred. Therefore it is to be said that the UKF is more robust than the EKF for larger sampling interval between measurements, for this type of orbit determination application.

\section{ACKNOWLEDGMENTS}

The authors wish to express their consideration to the Instituto Nacional de Pesquisas Espaciais (INPE) that kindly provided everything necessary for this paper to be developed. The authors are also grateful to Fundação de Amparo à Pesquisa do Estado de São Paulo (FAPESP) for the support, under contracts No. 2013/00960-4, No. 2012/21023-6; to CAPES; and to CNPq.

\section{REFERENCES}

Brown, R. G. and Hwang, P. Y. C., 1984, A Kalman filter approach to precision GPS geodesy, Navigation: Global Positioning System, Vol. II, pp. 155-166.

Brown, R. G. and Hwang, P. Y. C., 1985, Introduction to random signals and applied Kalman filtering, 3rd ed. New York: John Wiley \& Sons. 502 p.

Chiaradia, A. P. M., Kuga, H. K. Kuga and Prado, A. F. B. A., 2003, Single frequency GPS measurements in real-time artificial satellite orbit determination, Acta Astronautica, Vol. 53, n. 2, pp. 123-133.

El-Sheimy, N., Shin, E-H. and Niu, X., 2006, Extended vs. Unscented Kalman Filters for Integrated GPS and MEMS Inertial. Available from: http://www.insidegnss.com/auto/0306\%20Kalman.pdf

Guan, T., 2013, Special cases of the three body problem, [online Colorado University database]. Available from: http://inside.mines.edu/ fs_home/tohno/teaching/PH505_2011/Paper_TianyuanGuan.pdf

Jose, J. M., 2009, Performance comparison of Extended and Unscented Kalman Filter implementation in INS-GPS integration, Ph.D thesis. Czech Technical University in Prague, Prague (in English). Available from: http://epubl.ltu.se/1653-0187/2009/095/LTUPB-EX-09095-SE.pdf

Julier, S. J. and Uhlmann, J. K., 1997, A new extension of the Kalman filter for nonlinear systems, International Symposium on Aerospace/ Defense Sensing, Simulation and Controls. SPIE.

Julier, S. J.and Uhlmann, J. K., 2004, Unscented filtering and nonlinear estimation. IEEE Transactions on Automatic Control, Vol. 92, n. 3, Mar 2004.

Kaula, W.M., 1966, Theory of Satellite Geodesy. Blasdell Pub. Co. Walthmam, Mass.

Maybeck, P.S., 1982, Stochastic models, estimation, and control. Vol. 2, Academic Press, NY.

Montenbruck, O. and Gill, E., 2001, Satellite orbits: models, methods, and applications, Springer-Verlag, Berlin Heidelberg NewYork. 369 p.

Pardal, P. C. P. M., Kuga, H. K. and Vilhena de Moraes, R., 2009a, A discussion related to orbit determination using nonlinear sigma point Kalman filter, Mathematical Problems in Engineering, v. 2009, 12 p. Hindawi Publishing Corporation, doi:10.1155/2009/140963.
Pardal, P. C. P. M., Kuga, H. K. and Vilhena de Moraes, R., 2009b, Non linear sigma point Kalman filter applied to orbit determination using GPS measurements, 22nd International Meeting of the Satellite Division of the Institute of Navigation (ION GNSS 2009), 22-25 Sept., Savannah, USA.

Pardal, P. C. P. M., Kuga, H. K. and Vilhena de Moraes, R., 2010, Comparing the extended and the sigma point Kalman filters for orbit determination modeling using GPS measurements,23rd International Meeting of the Satellite Division of the Institute of Navigation (ION GNSS 2010), 21-24 Sept., Portland, USA.

Pardal, P. C. P. M., Real time orbit determination through non linear sigma-point Kalman filter, 2011, Ph.D thesis, National Institute for Space Research (INPE), São José dos Campos. (In Portuguese).

Pardal, P. C. P. M., Kuga, H. K.and Vilhena de Moraes, R., 2011, Robustness assessment between sigma point and extended Kalman filter for orbit determination,. Journal of Aerospace Engineering, Sciences and Applications, v. III, pp. 35-44. doi: 10.7446/ jaesa.0303.04

Prado, A. F. B. A. and Kuga, H. K. (Eds.), 2001, Space Technology Fundaments, São José dos Campos: INPE. 220 p. (In Portuguese).

Soken, H. E. and Hajiyev, C., 2011, REKF and RUKF Development for Pico Satellite Attitude Estimation in the Presence of Measurement Faults, 5th International Conference on Recent Advances in Space Technologies (RAST), 9-11 June 2011. pp. 891-896. doi: 10.1109/ RAST.2011.5966972

van der Merwe, R., 2004, Sigma-Point Kalman filters for probabilistic inference in dynamic state-space models, Ph.D thesis. Oregon Health \& Science University, Portland.

van der Merwe, R., Wan, E.A. and Julier, S.J., 2004, Sigma-point Kalman filters for nonlinear estimation and sensor-fusion applications to integrated navigation, AIAA Guidance, Navigation, and Control Conference and Exhibit, 16-19 Aug. 2004, Rhode Island.

Wan, E. A. and van der Merwe, R., 2001, The unscented Kalman filter, Kalman Filtering and Neural Networks. Haykins, S. [Ed.], John Wiley \& Sons, New York, Chap. 7. 